\title{
The New Combined Method of the Generation of a 3d Dense Map of Evironment based on History of Camera Positions and the Robot's Movements
}

\author{
Aleksander Vokhmintcev ${ }^{1,2}$, Michail Timchenko ${ }^{3}$ \\ 1 "Intelligent information technologies" Research laboratory, Chelyabinsk State \\ University, 129 Br. Kashirinyh, 454001, Chelyabinsk, Russia, vav@csu.ru \\ ${ }^{2}$ Institute of Digital economy, Ugra State University, 16 Chekhova street, 628012, \\ Khanty-Mansiysk, Russia vav@ugrasu.ru \\ ${ }^{3}$ School of Electrical Engineering and Computer Science, South Ural State \\ University, 76 Lenin prospect, 454080, Chelyabinsk, Russia \\ timchenkoms@susu.ru
}

Abstract: A combined solution is proposed for solving the point-plane variational problem in a closed-form based on fusion visual features and $3 d$ point clouds. An accurate method for reconstructing $3 d$ scene is introduced, and the closed-form solutions of registration task for orthogonal transformation are presented. This method is used to reconstruct a threedimensional model of the environment from a set of images and depth map. The given method is used to match and register point clouds with an arbitrary spatial resolution and an arbitrary scale with respect to each other. The suggested method improves the convergence and accuracy of reconstruction methods for dynamic, bulky scenes.

Keywords: $3 d$ mapping; registration problem; three-dimensional map; feature-matching; iterative closest point algorithm

\section{Introduction}

In order to promptly involve in the development of oil reserves of the YNAO in the conditions of high laboriousness of mapping filtering channels and large amounts of seismic data, it is necessary to develop information technology for mapping filtering channels based on machine learning methods and artificial intelligence. In this paper, we consider an approach that allows us to automate the procedure for selecting fracture zones in $2 \mathrm{D}$ images and $3 \mathrm{D}$ models and a present new method for the identification of neotectonic structures from digital maps of 
the terrain based on a $3 \mathrm{~d}$ reconstruction methods of environment and SLAM (Simultaneous Location and Mapping). This method is used for reconstruction of seismic tracks in a three-dimensional seismic cube. We will use the suggested approach to monitor the mineral deposits in the region using a mobile robot platform. The development of an adaptive dynamic system for robot mapping and navigation in real-time is one of the key tasks in modern robotics and machine vision. Over the last decade numerous methods of SLAM have been suggested [1]. The fast methods of SLAM and the methods based on orienting points for localization in an unknown environment were the first to be worked out. Subsequently, the main direction of investigations was connected with the development of intellectual SLAM methods which use various multi-sensors and Kalman filtering to estimate the motion trajectory of a robotic system in unknown space [2]. To date, the following solutions to the SLAM problem are known: graphic SLAM, particle filter SLAM, Extended Kalman filter (EKF), and visual SLAM [3]. In addition to these approaches of the SLAM the following methods can be used: extreme methods of navigation based on comparison of two consecutive scans by optimizing the cross-correlation function; the method of recursive filtering; method Normal Distributions Transform is a transformation of normal distributions. The disadvantages of this algorithm should include the strong dependence of convergence from the initial approximations and discretization of space.

The performance of SLAM methods directly depends on the accuracy of the $3 \mathrm{~d}$ mapping method which allows to create shape model for example based on the registration task of the $3 \mathrm{~d}$ point clouds obtained from depth sensors. The most popular registration method is the Iterative Closest Point (ICP) algorithm [4]. A key stage in the ICP algorithm is a search for an orthogonal or affine transformation that imposes two point clouds with a given matching of points in the best mean. The most commonly used methods for searching correspondence between a pair of $3 \mathrm{~d}$ clouds are ICP algorithm with point-to-point metric and ICP algorithm with point-to-plane metric: the second metric is superior to the first metric in terms of accuracy and convergence [5]. The point-to-point task can be solved by applying the Levenberg-Marquardt algorithm. It has typical shortcomings of iterative methods, namely, the accuracy of the registration depends on the chosen initial approximation [6]. In the class of orthogonal transformations, a closed-form solution of the point-to-point task was produced with the help of quaternions in or orthogonal matrices in [7]. Horn's method was used in [8] to formulate an ICP algorithm in the point-to-plane version. For the variational problem of the point-plane for the class of affine transformations, the exact solution was proposed [9]. An approximate solution of the point-plane problem for the class of orthogonal transformations was obtained in [10]. A registration algorithm ICP of point clouds of no rigid objects was proposed [11]. The ICP algorithm is characterized by two main problems: first, the algorithm does not use the local surface shape around each point, and secondly, the search of nearby points has a high computational complexity $[12,13]$. The approach based 
on point-to-plane has more constraints of the structure of the reconstructed scene [14]. The ICP algorithm using the point-to-plane metric has a poor convergence for scenes with a small number of geometric constraints [15]. The convergence of the ICP algorithm may be considerably improved [16]. Since modern RGB-D cameras provide color and depth information, a data fusion from two sources are desirable. We suggest applying visual (color) features to significantly improve the initial point of the ICP algorithm, and the alignment between key-frames is computed by joint optimization of appearance and shape matches. [17]. The most successful feature descriptors such as SIFT (Scale Invariant Feature Transform) [18] and SURF (Speeded-Up Robust Features) [19] are robust to scale and viewpoint changes. However, other visual features could be also useful for localization and global optimization. For example, histograms of oriented gradients (HOGs) possess attractive invariance to viewpoint changes [20]. Within the framework of this project a closed-form solution of the point-plane problem for the class of orthogonal transformations will be found, and an iterative registration method was suggested. The fusion ICP method is used for reconstruction of seismic tracks in a three-dimensional seismic cube.

The paper is organized as follows. In Section 2, the proposed effective fusion algorithm for registration $3 \mathrm{~d}$ data is presented. In Section 3, the robot mapping algorithm is considered. Computer simulation results are provided in Section 4. Section 5 summarizes our conclusions.

\section{Fusion Iterative Closest Point Algorithm}

This project has two main objectives:

- the development of new accurate registration methods for reconstruction of three-dimensional maps in an unknown dynamic environment;

- the development of a new path planning method of a mobile robot in an unknown dynamic space which have theoretically approved estimates of computational complexity.

Modern practical solutions of a SLAM task for large-scale scenes are based on metric-topological and visual approaches. In this case, the SLAM task can be described in the form of the following scheme:

- the local reconstruction of a map using metrics to assess a trajectory and the creation of local maps;

- the recognition of places in order to solve the problem of "loop closure" or to fulfill the task of relocalization;

- the optimization of a constructed map and global optimization. 
Let us define some requirements for input data and for objects of a scene:

- the robot determines its position in an environment relative to the target point;

- $\quad$ objects on a scene have a rigid shape (rigid objects);

- the degree of freedom of a robot' movement is $6 \mathrm{D}$;

- it is necessary to consider constructive and dynamic limitations of the robot for the path planning;

- no odometrical information is used;

- no plan of the environment for a mobile robot is preset;

- the environment contains obstacles, including dynamic ones;

- the accuracy of the evaluation of a trajectory is determined by a type of sensors employed.

The algorithm for constructing a three-dimensional map of a surface consists of the following basic steps:

Step 1. Measure a depth map and compute a normalized surface.

Step 2. Estimate the camera position by applying the ICP method between the predicted and measured surfaces.

Step 3. Refine the global map of the surface by incorporating the measured surface into the map constructed at the preceding steps.

Step 4. Predict the surface at the next step by applying modified Kalman filtering. Go to Step 2.

A scheme of the proposed method is presented on Fig. 1.

Suppose that $X=\left\{x_{1}, \ldots, x_{n}\right\}$ and $Y=\left\{\mathrm{y}_{1}, \ldots, \mathrm{y}_{m}\right\}$ be an initial point cloud and a target point cloud in $\mathrm{R}^{3}$ accordingly. Then each iteration in the variational problem of the classical ICP algorithm for an arbitrary affine transformation can be represented as follows:

$$
R S x_{i}+T
$$

where $R$ is a rotation matrix, $T$ is a translation vector, $i=1, \ldots, n$., $S$ is a scaling matrix.

The matching function becomes [5]:

$$
E(R, S, T)=\frac{1}{N} \sum_{i=1}^{N}\left\|\left(R S x_{i}+T\right)-y_{i}\right\|^{2},
$$

where $l_{\mathrm{m}}$ is the number of corresponding points pairs. 


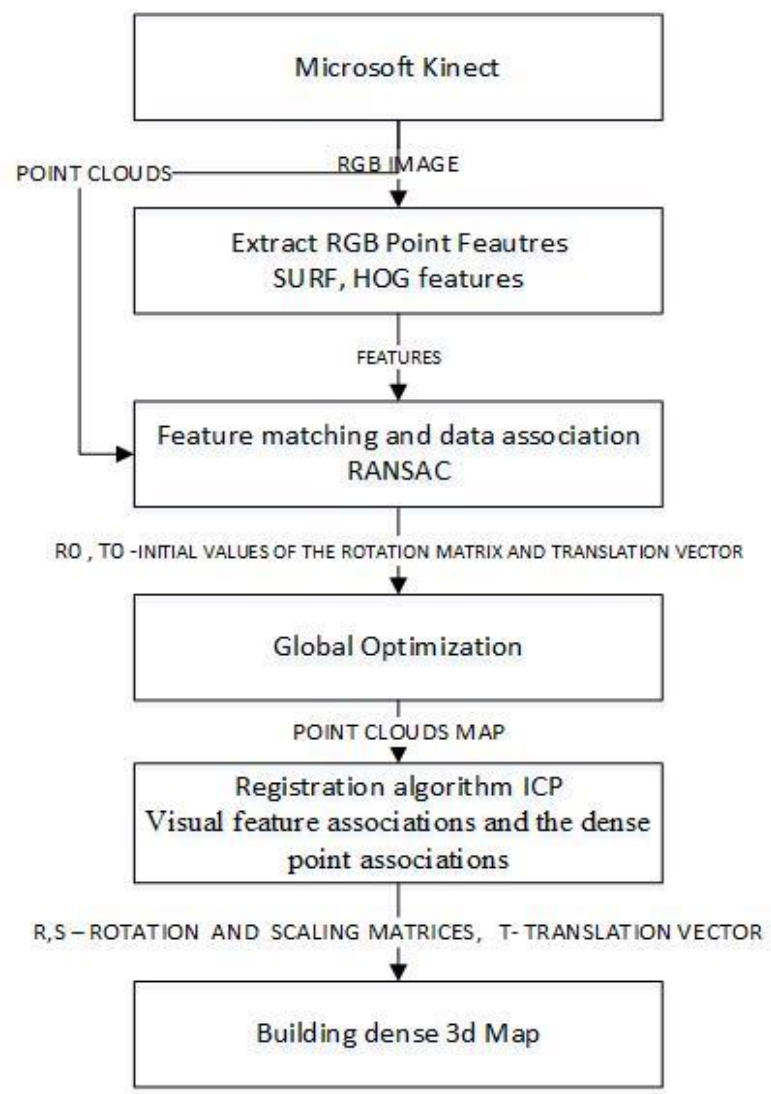

Figure 1

A scheme of the fusion registration method combining visual features and depth information

If (2) has minimum it will satisfy the following equation $\frac{d E(T)}{d T}=0$.

$\frac{d E(T)}{d T}=\frac{2 S}{N} \sum_{i=1}^{N}\left(\left(R S x_{i}+t\right)-y_{i}\right)=0$

So, we can make the following conclusion $T=\frac{1}{N} \sum_{i=1}^{N} y_{i}-\frac{1}{N} \sum_{i=1}^{N} R S x_{i}$. Therefore, matching function is as follows in order to achieve the minimum $E(R, S)=\frac{1}{N} \sum_{i=1}^{N}\left\|R S\left(x_{i}-\frac{1}{N} \sum_{i=1}^{N} x_{i}\right)-\left(y_{i}-\frac{1}{N} \sum_{i=1}^{N} y_{i}\right)\right\|^{2}$ 
Let $a_{i}=\left(x_{i}-\frac{1}{N} \sum_{i=1}^{N} x_{i}\right), b_{i}=\left(y_{i}-\frac{1}{N} \sum_{i=1}^{N} y_{i}\right)$, taking into account this equation, the matching function can be represented as follows:

$$
E(R, S)=\frac{1}{N} \sum_{i=1}^{N}\left\|R S a_{i}-b_{i}\right\|^{2}
$$

Then

$$
E(R, S)=\frac{1}{l_{m}}\left(\sum_{i=1}^{l_{m}} a_{i}^{T} S^{2} a_{i}-2 \sum_{i=1}^{l_{m}} b_{i}^{T} R S a_{i}+\sum_{i=1}^{l_{m}} b_{i}^{T} b_{i}\right) .
$$

Then we find out the minimum of this matching function:

$$
\frac{\mathrm{dE}(\mathrm{R}, \mathrm{S})}{\mathrm{dR}}=0 \frac{\mathrm{dE}(\mathrm{R}, \mathrm{S})}{\mathrm{dS}}=0
$$

Then we can get scale matrix from (7):

$$
\frac{d E(R, S)}{d S}=2 \sum_{i=1}^{l_{m}} a_{i}^{T} S E_{J} a_{i}-2 \sum_{i=1}^{l_{m}} b_{i}^{T} R S E_{J} a_{i}=0
$$

where $E_{j}=(0, \cdots, 0,1,0, \cdots, 0), j=1,2,3$ is s-diagonal scale matrix. Then the scale matrix can be computed as follows:

$$
S_{j}=\frac{\sum_{i=1}^{l_{m}} b_{i}^{T} R E_{j} a_{i}}{\sum_{i=1}^{l_{m}} a_{i}^{T} R E_{j} a_{i}} .
$$

Let $\mathrm{A}$ is the transformation matrix including the components of the rotation matrix $\mathrm{R}$; and $\mathrm{T}$ is the translation vector. Let $J(A, T)$ be the following function:

$$
J(A, T)=\sum_{i=1}^{n} \square A x_{i}+T-y_{i} \square^{2}
$$

Then new project solutions to $3 \mathrm{~d}$ total variation regularization will be obtained [21]. The function $J(A, T)$ can be rewritten:

$$
\begin{aligned}
& J(A)=\sum_{i=1}^{n}\left(<A p_{i}-q_{i}, n_{i}>\right)^{2}=\sum_{i=1}^{n}\left(<A p_{i}, n_{i}>-<q_{i}, n_{i}>\right)^{2}= \\
& =\sum_{i=1}^{n}<A p_{i}, n_{i}>^{2}-2<A p_{i}, n_{i}><q_{i}, n_{i}>+<q_{i}, n_{i}>^{2}
\end{aligned}
$$

where $p_{i}$ is a point from the point cloud $P, n_{i}$ is the unitary normal for the tangent plane $T\left(q_{i}\right)$. 
Let us consider the partial derivatives with respect to $a_{i j}$ :

$$
\frac{\partial J}{\partial a_{i j}} J(A, T)=\sum_{k=1}^{n} 2<A p^{k}, n^{k}>p_{j}^{k}, n_{i}^{k}-2 p_{j}^{k}, n_{i}^{k}<q^{k}, n^{k}>=0,
$$

where $i, j=1, \ldots, 3, k=1, \ldots, n$.

$$
\sum_{i=1}^{n}<A p^{k}, n^{k}>p_{j}^{k}, n_{i}^{k}-p_{j}^{k}, n_{i}^{k}<q^{k}, n^{k}>=\sum_{i=1}^{n}<A p^{k}, n^{k}>p_{j}^{k}, n_{i}^{k}-\sum_{i=1}^{n} p_{j}^{k}, n_{i}^{k}<q^{k}, n^{k}>=0
$$

The partial sum $\sum_{k=1}^{n} p_{j}^{k}, n_{i}^{k}<q^{k}, n^{k}>$ is a constant with respect to $A$ :

$$
\sum_{k=1}^{n} p_{j}^{k}, n_{i}^{k}<q^{k}, n^{k}>=c_{i j}, i, j=1, \ldots, 3
$$

Taking into account Equations 14 can be rewritten as

$$
\sum_{k=1}^{n} p_{j}^{k}, n_{i}^{k} \operatorname{tr}\left(A \sqcap(\mathrm{PN})^{k}\right)=c_{i j}, i, j=1, \ldots, 3 .
$$

Let $\widehat{M}$ is the transformation matrix including the components of the rotation matrix $\mathrm{R}$; and $\mathrm{T}$ is the translation vector. In the first iteration, a matrix $\widehat{M}$ that is generated by the matrix $\mathrm{A}$ and vector $\mathrm{T}$ can be initialized by the visual RANSAC transformation. In the proposed formulation of the problem we find a solution of variation problem based on the combination of data on feature points (the color scenes), and a dense three-dimensional point cloud (depth data). The considered functionals are composed of terms that measure the average of the squares of the distances to visual-associated characteristic points with the normalizing factor (the variation of metric characteristics of a function of two variables), terms that measure the average of the squares of the distances for a dense point cloud based on metrics point-to-plane. The solution of the variation problem obtained using various iterative methods. Next, we minimize the alignment error of both visual feature and dense point associations.

Let $\mathrm{A}_{\mathrm{f}}$ is a set of matches consisting of pairs of singular points in the image that are related to the best transformation; similarly; $A_{d}$ is a set of matches in the cloud of points associated with the best transformation; $\alpha$ is an empirically chosen weighting coefficient; $\mathrm{w}_{\mathrm{i}}$ and $\mathrm{w}_{\mathrm{j}}$ - weighting coefficients for features and depth data respectively. The joint error function is given as 


$$
\mathrm{E}(\mathrm{R}, \mathrm{T})=\operatorname{argmin}_{\hat{\mathrm{M}}}\left[\begin{array}{c}
\alpha \frac{1}{\mathrm{~W}}\left(\frac{1}{\left|\mathrm{~A}_{\mathrm{f}}\right|} \sum_{\mathrm{i}} \mathrm{W}_{\mathrm{i}}\left|\hat{\mathrm{M}} \mathrm{F}_{\mathrm{s}}^{\mathrm{i}}-\mathrm{F}_{\mathrm{d}}^{\mathrm{i}}\right|^{2}\right)+ \\
(1-\alpha) \frac{1}{\mathrm{~W}}\left(\frac{1}{\left|\mathrm{~A}_{\mathrm{d}}\right|} \sum_{\mathrm{j}} \mathrm{w}_{\mathrm{j}}\left|\left(\left(\hat{\mathrm{M}} \mathrm{p}_{\mathrm{j}}-q_{\mathrm{j}}\right) \mathrm{n}_{\mathrm{j}}\right)\right|^{2}\right)
\end{array}\right] .
$$

The functions $F_{S}^{I}$ and $F_{d}^{I}$ provide the projection of a feature point from its Euclidean $3 \mathrm{~d}$ position into the image space of the camera $[2,22]$.

\section{Robot Mapping Algorithm}

In this project, we use approach to the implementation of the task is based on the application of Extended Kalman Filter, EKF. We use the term visual feature and term semantic landmark synonymously. The robot platform is equipped with a multi-sensor cameras (Kinect and Beward camera) which take measurements of the relative location between any semantic landmark and the robot platform itself. The absolute locations of the semantic tags are not available. The implementation of the suggested visual SLAM algorithm uses nonlinear kinetic models of robot platform and non-linear asynchronous observation models in controlled/ uncontrolled indoor conditions). The state of the system of interest consists of the position and orientation of the robot platform together with the position of all semantic tags. The general algorithm of $3 \mathrm{~d}$ mapping is shown in Fig. 2.

The updating of a history of camera positions and the robot's movements, as well as the configuration of surveyed characteristics are carried out at a certain interval of time. As a rule, data is added not at every step, but in case the position of the camera (the robot) has significantly changed, for example, when a considerable displacement or rotation has taken place as compared to the previous memorized position. A mathematical model of the scene was saved in the form of a graph the vertices of which correspond to certain moments of time. In the present work, a new combined adaptive method was developed for the generating a threedimensional combined dense map of an accessible environment and determining the position of the robot in a relative coordinate system based on the history of camera positions, on symbolic (semantic) tags, on the robot's movements and on the matching of obtained three-dimensional depth maps which account for the accuracy of their superimposition, as well as geometric relationships between various images of one scene [23]. 


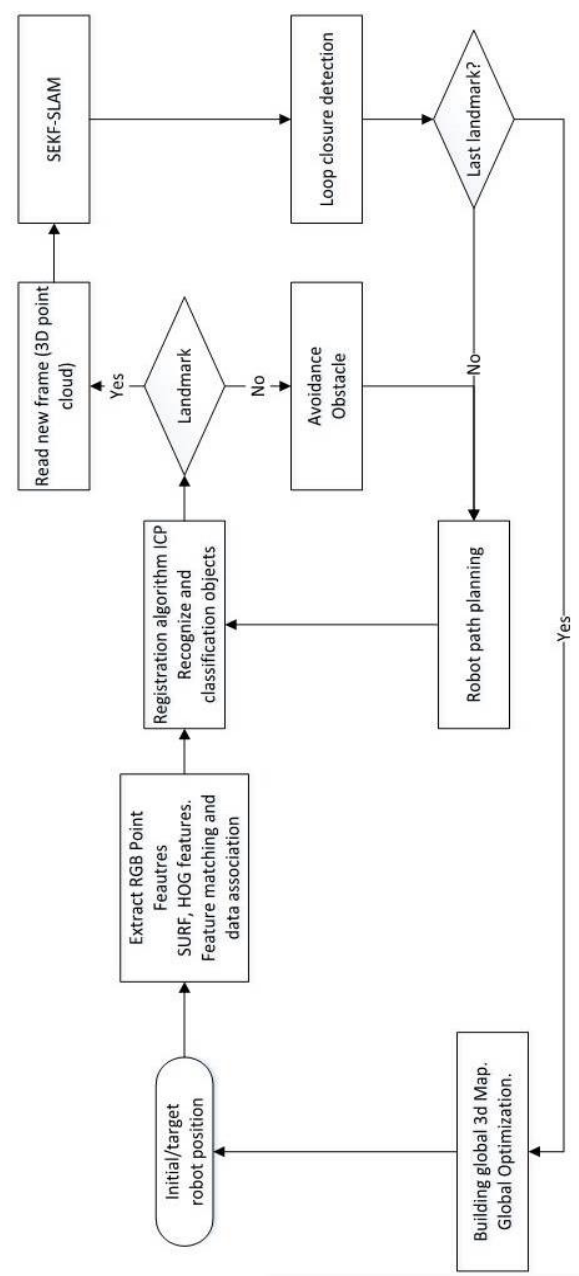

Figure 2

A block diagram of the proposed $3 \mathrm{~d}$ mapping method

\section{Computer Simulation}

In this section, computer simulation results are presented and discussed. We used NYU Depth Dataset for computer simulation. In this section, the proposed fusion registration method is compared with the Fast ICP and point-to-plane ICP algorithms. The performance of the registration method is shown in Table 1 compared with that of the tested algorithms. The second row shows the 
performance of the algorithms in terms of computational complexity. Each row has two values: first value show matching accuracy (\%), the second value shows time in sec. Fig. 3 shows the performance of the tested algorithms in terms of mean square error. One can observe that the fusion registration method has the best performance.

Table 1

Matching accuracy (\%) and computational complexity (sec) depending on rotation angle

\begin{tabular}{|c|c|c|c|c|c|c|}
\hline \multirow{2}{*}{ Algorithms } & \multicolumn{7}{|c|}{ Rotation angle (degrees) } \\
\cline { 2 - 7 } & 5 & 10 & 15 & 20 & 25 & 30 \\
\hline Fast ICP & $91 / 0,06$ & $80 / 0,1$ & $73 / 0,11$ & $66 / 0,13$ & $56 / 0,15$ & $47 / 0,17$ \\
\hline ICP & $97 / 1,56$ & $95 / 1,86$ & $91 / 2,34$ & $86 / 3,8$ & $81 / 4,17$ & $78 / 6,78$ \\
\hline $\begin{array}{c}\text { Fusion } \\
\text { algorithm }\end{array}$ & $98 / 1,45$ & $99 / 1,78$ & $98 / 2,4$ & $96 / 2,95$ & $91 / 3,2$ & $92 / 3,63$ \\
\hline
\end{tabular}

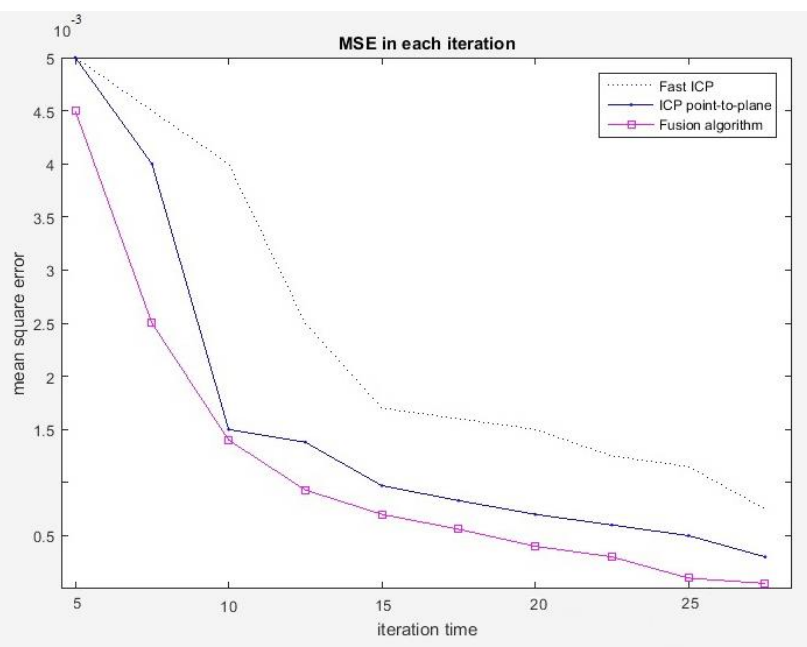

Figure 3

The performance of the algorithms in terms of mean square error

To process visual scene characteristics, we use an image-matching algorithm based on the recursive calculation of oriented gradient histograms for several circular sliding windows and a pyramidal image decomposition [20, 24]. The algorithm yields initial values for a fusion registration algorithm. State of art image matching algorithms (SIFT, SURF, ORB) and suggested algorithm were tested in a series of different experiments:

- transformation "image rotation in the scene plane" (Table 2);

- $\quad$ transformation "rotation outside the scene plane" (Table 3);

- $\quad$ conversion of scale changes (Table 4); 
The matching algorithms were probed in different conditions such as in-plane/outof-plane rotations and scaling changes. The performance matching algorithms were estimated in terms of the number of accurate matches. The suggested algorithm gives a more stable matching performance than other algorithms for out-of-plane rotations (Table 3) and small scaling (Table 4). In the future, we plan to test the suggested registration method using Netherlands Dataset: A New Public Dataset for Machine Learning in Seismic Interpretation.

Table 2

Accuracy of matching (in \%) of various algorithms vs.in-plane rotation angle

\begin{tabular}{|l|c|c|c|c|c|c|}
\hline \multirow{2}{*}{ Matching algorithm } & \multicolumn{7}{|c|}{ Angle of rotation, } \\
\cline { 2 - 7 } & 45 & 90 & 135 & 180 & 225 & 270 \\
\hline SIFT & 100 & 98 & 99 & 98 & 97 & 95 \\
\hline SURF & 74 & 69 & 74 & 69 & 74 & 69 \\
\hline ORB & 87 & 85 & 83 & 86 & 85 & 87 \\
\hline Proposed algorithm & 100 & 98 & 96 & 98 & 97 & 95 \\
\hline
\end{tabular}

Table 3

Accuracy of matching (in \%) of various algorithms vs. off-plane rotation angle

\begin{tabular}{|l|c|c|c|c|c|c|}
\hline \multirow{2}{*}{ Matching algorithm } & \multicolumn{7}{|c|}{ Angle of rotation, $\square$} \\
\cline { 2 - 7 } & 5 & 10 & 15 & 20 & 25 & 30 \\
\hline SIFT & 98 & 91 & 78 & 64 & 58 & 47 \\
\hline SURF & 82 & 77 & 64 & 55 & 38 & 29 \\
\hline ORB & 96 & 84 & 77 & 61 & 58 & 54 \\
\hline Proposed algorithm & 83 & 78 & 74 & 72 & 76 & 72 \\
\hline
\end{tabular}

Table 4

Accuracy of matching (in \%) of various algorithms vs. small scaling changes

\begin{tabular}{|l|c|c|c|c|c|}
\hline \multirow{2}{*}{ Matching algorithm } & \multicolumn{5}{|c|}{ Scaling factor } \\
\cline { 2 - 6 } & $0.8 \mathrm{X}$ & $0.9 \mathrm{X}$ & $1.0 \mathrm{X}$ & $1.1 \mathrm{X}$ & $1.2 \mathrm{X}$ \\
\hline SIFT & 92 & 95 & 100 & 98 & 91 \\
\hline SURF & 79 & 90 & 99 & 97 & 92 \\
\hline ORB & 78 & 79 & 90 & 83 & 89 \\
\hline Proposed algorithm & 84 & 94 & 100 & 99 & 91 \\
\hline
\end{tabular}

\section{Conclusions}

In this paper, the accurate method for reconstructing $3 \mathrm{~d}$ scene and the closed-form solutions of registration task for orthogonal transformation combining visual features and the depth information were proposed. The obtained results were illustrated with the help of computer simulation. In the proposed formulation of the problem we find a solution of variational task point-to-plane based on the 
combination of data on feature points (the color scenes), and a dense threedimensional point cloud (depth data). The considered functionals are composed of terms that measure the average of the squares of the distances to visual-associated characteristic points with the normalizing factor (the variation of metric characteristics of a function of two variables), the terms that measure the average of the squares of the distances for a dense point cloud based on a metrics point-toplane. The given method is used to match and register a point clouds with an arbitrary spatial resolution and an arbitrary scale with respect to each other and provide accurate estimates for complex dynamic large-scale scenes. Within the framework of the project, the variational problem of the ICP algorithm is extended to a class of affine transformations. The computational experiments carried out showed a significant increase in the accuracy of the suggested registration method. The results of the project have shown that the use of an approximate $3 \mathrm{~d}$ map of the surrounding environment significantly improves the quality of recognition and localization of objects in dynamic, bulky scenes, especially with partial or complete occlusion of an object by other scene objects.

\section{Acknowledgement}

The reported study was funded by RFBR and Yamalo-Nenets Autonomous Okrugr, project number 19-47-890001.

\section{References}

[1] C. Hertzberg, R. Wagner and O. Birbach "Experiences in building a visual slam system from open source components", In. Proc. IEEE International Conference on Robotics and Automation, pp. 2644-2651, Shanghai, China, May 09-13, 2011

[2] P. Henry, M. Krainin and E. Herbst "RGB-D mapping: Using depth cameras for dense 3D modeling of indoor environments", In. Proc. $12^{\text {th }}$ International Symposium on Experimental Robotics, pp. 477-491, Delhi, India, Dec 18-21, 2014

[3] F. Endres, J. Hess, N. Engelhard and J. Sturm "An evaluation of the RGBD SLAM system", In. Proc. IEEE International Conference on Robotics and Automation, pp. 1691-1696, Taipei, Taiwan, Oct 18-22, 2012

[4] P. Besl and N. McKay "A Method for Registration of 3-D Shapes Trans", IEEE Transaction on Pattern Analysis and Machine Intelligence, IEEE, Vol. 14(2) pp. 239-256, 1992

[5] S. Ying, J. Peng, S. Du and H. Qiao "A Scale Stretch Method Based on ICP for 3D Data Registration", IEEE Transactions on Automation Science and Engineering, IEEE, Vol. 6(3) pp. 559-565, 2009

[6] G. Tam, Z.-Q. Cheng, Y.-K. Lai, F. Langbein, Y. Liu, D. Marshall, R. Martin, X.-F. Sun, and P. Rosin "Registration of 3D point clouds and meshes: A survey from rigid to nonrigid", IEEE Transactions on Visualization and Computer Graphics, IEEE, Vol. 19 (7), pp. 1199-1217, 2013 
[7] B. Horn B., H. Hilden and S. Negahdaripour S. "Closed-form Solution of Absolute Orientation Using Orthonormal Matrices", Journal of the Optical Society of America, Vol. 5 (7), pp. 1127-1135, 1988

[8] K. L. Low "Linear least-squares optimization for point-to-plane ICP surface registration", Technical Report TR04-004, Department of Computer Science, University of North Carolina at Chapel Hill, 2004

[9] S. Du, N. Zheng, S. Ying and J. Liu "Affine iterative closest point algorithm for point set registration", Pattern Recognition Letters, Elsevier, Vol. 31(9), pp. 791-799, 2010

[10] K. Khoshelham "Closed-form solutions for estimating a rigid motion from plane correspondences extracted from point clouds", ISPRS Journal of Photogrammetry and Remote Sensing, Elsevier, Vol. 114, pp. 78-91, 2016

[11] S. Cheng, I. Marras and S. Zafeiriou "Active nonrigid ICP algorithm", In Proc. $11^{\text {th }}$ IEEE International Conference and Workshops on Automatic Face and Gesture Recognition, pp. 1-8, Ljubljana, Slovenia, May 4-8, 2015

[12] Y. Chen and G. Medioni "Object Modeling by Registration of Multiple Range Images", In. Proc. IEEE International Conference on Robotics and Automation, Vol. 10(3) pp. 145-155, Sacramento,USA, April 17-19, 1991

[13] C. Langis, M. Greenspan and G. Godin "The parallel Iterative closest point algorithm", In. Proc. IEEE Third International Conference 3-D Digital Imaging and Modeling, pp. 195-204, Quebec City, Canada, 28 May-1 June, 2001

[14] S. Thrun, W. Burgard and D. Fox "A real-time algorithm for mobile robot mapping with applications to multi-robot and 3D mapping", In. Proc. IEEE International Conference on Robotics and Automation, pp. 321-328, San Francisco, USA, April 24-28, 2000

[15] A. Segal, D. Haehnel and S. Thrun "Generalized-ICP", In. Proc. of Robotics: Science and Systems, Vol. 2(4) pp. 645-653, Seattle, USA, June 6-9, 2009

[16] S. Rusinkiewicz and M. Levoy "Efficient variants of the ICP algorithm", In Proc. International Conference on 3-D Digital Imaging and Modeling (3DIM) pp. 145-152, Quebec City, Canada, May 28-June 1, 2001

[17] M. Fischler and R. Bolles "Random Sample Consensus: A Paradigm for Model Fitting with Applications to Image Analysis and Automated Cartography", Graphics and Image Processing, pp. 381-395, 1981

[18] D. G. Lowe "Object recognition from local scale invariant features", In. Proc. $7^{\text {th }}$ International conference on Computer Vision, Vol. 2, pp. 11501157, Kerkyra, Greece, September 20-27, 1999 
[19] H. Bay, A. Ess, T. Tuytelaars and L. Van Gool "SURF: Speeded Up Robust Features", Computer Vision and Image Understanding, Journal Elsevier, Vol. 110, pp. 346-359, 2008

[20] D. Miramontes-Jaramillo, V. Kober, V. H. Diaz-Ramirez and V. Karnaukhov V. "A novel Image Matching Algorithm Based on Sliding Histograms of Oriented Gradients", Journal of Communications Technology and Electronics, Springer, Vol. 59(12) pp. 1446-1450, 2014

[21] A. Vokhmintcev, A. Melnikov, K. Mironov, V. Burlutskiy "Reconstruction of three-dimensional map based on closed form solution of variational problem of multi-sensor data registration", Doklady Mathematics, Springer, Vol. 99 (1) pp. 108-112, 2019

[22] A. Vokhmintsev and K. Yakovlev "A Real-time Algorithm for Mobile Robot Mapping Based on Rotation invariant Descriptors and ICP", In Proc. $5^{\text {th }}$ Analysis of Images, Social Networks and Texts, Communications in Computer and Information Science, Vol. 661, pp. 357-369, Ykaterinburg, Russia, April 7-9, 2017

[23] I. Sochenkov and A. Vokhmintsev "Visual duplicates image search for a non-cooperative person recognition at a distance", Procedia Engineering, Elsevier B. V., Vol. 129, pp. 440-445, 2015

[24] A. Vokhmintcev, I. Sochenkov, V. Kuznetsov V. and D. Tikhonkikh "Face recognition based on matching algorithm with recursive calculation of local oriented gradient histogram”, Doklady Mathematics, Springer, Vol. 93(1) pp. 37-41, 2016 